\title{
A current view on inflammation
}

\section{Evolving mechanistic and conceptual understanding of inflammation drives insight into human disease and new approaches for therapy.}

n nflammation is generally defined as a response to stimulation by invading pathogens or endogenous signals such as damaged cells that results in tissue repair or sometimes pathology, when the response goes unchecked. However, understanding of the mechanisms, context and role of inflammation during physiological immune responses and pathology is constantly evolving. Recent advances have been driven by increased understanding of the commensal microbiota, immunometabolism and cancer and by technical advances such as single-cell analysis and high-throughput epigenetic, transcriptional and proteomic profiling of cells. In this Nature Immunology Focus (http://www.nature. $\mathrm{com} / \mathrm{ni} /$ focus/inflammation2017.html), these various facets of inflammation are discussed.

Each tissue exhibits distinct characteristics of inflammation as a result of general and local molecular, immunological and physiological processes. A Comment authored by Netea and 31 other experts on various aspects of inflammation provides a brief, up-to-date guide to the basic characteristics of inflammation in various tissues and its manifestations in the context of human disease.

Study of a particular subset of human diseases, the autoinflammatory syndromes, has brought invaluable insight into the intrinsic link between inflammation and pathology in humans. The concept of autoinflammation was proposed almost two decades ago to delineate a group of diseases that manifest various forms of systemic inflammation involving myeloid cells but lack aspects of autoimmune disease, such as high titers of autoantibodies and the accumulation of antigen-specific T cells. Kastner and colleagues review monogenic inflammatory diseases caused by mechanisms as varied as dysregulated inflammasomes, perturbations in ubiquitination, protein folding or complement activation. The authors describe how a combination of constantly evolving, unbiased genomic methods and clinical medicine has allowed insight into physiologically relevant pathways of inflammation and has revealed new treatment options.

Kroemer and colleagues offer a fresh perspective on another human disease long acknowledged as being associated with inflammation. Cancer occurs as the clinical manifestation of stochastic molecular events linked to cellular turnover and mutagenesis, as well as to avoidable environmental and life-style factors. The authors discuss the effects of one of the most controllable life-style factors-diet-on human health and analyze the complex connections among nutrition, inflammation and cancer. As the authors point out, although the link between diet and cancer is not as linear as that between tobacco use and lung cancer, strong immunomodulatory effects of diet have been found, in particular in the context of overeating, fasting and the composition of food. Obesity is linked to chronic inflammation though a variety of mechanisms, including the inhibition of autophagy and acceleration of processes associated with aging, while fasting has considerable anti-inflammatory effects. The authors discuss how various diets and nutritional interventions can affect carcinogenesis and the response to cancer therapy.

Blander and colleagues review the role of another important contributor to inflammation, homeostasis and health: the intestinal microbiota. Studies in this area are moving past cataloging of the commensal community into elucidating their contribution to physiology of the host and defining the so-called 'immune-relevant' microbiome. Important correlations between specific components of the microbiota and disease have been documented, ranging from autoimmune diseases to neurodegenerative disorders, with chronic inflammation as the driver of many of these conditions. This Review discusses recent insights in the molecular underpinnings of host-microbiota interactions that influence inflammation in the intestine or at distal sites. Interconnected factors such as long-term dietary habits, the bio-ecology of the intestinal environment and the production of bacterial metabolites affect mucosal and systemic immunity as well as susceptibility to autoimmunity and infection and the response to vaccination or cancer therapy.

Within the physiological parameters of a protective immune response, inflammation is essential for efficient immunity, which includes tissue healing and return to homeostasis. Physiological inflammation is selflimiting and self-regulated. Beyaert, Karin and colleagues look at the complex, multilayered negative auto-regulatory loops that restrict and resolve inflammation, with a focus on the post-transcriptional and nondegradative ubiquitin-mediated regulation of signaling involving the transcription factor NF- $\mathrm{KB}$ and the NLRP3 inflammasome, two archetypal drivers of the inflammatory response.

Molecular and conceptual understanding of inflammation will probably keep evolving at a fast pace. Research into new and exciting fields, such as aging and senescence, neurological-immunological interactions, cellular metabolism and epigenetics, should offer new insights and bring more conceptual shifts. Such research will allow efficient and specific intervention into physiological pathways of inflammation to generate novel therapeutic approaches for old diseases and modern health challenges. 